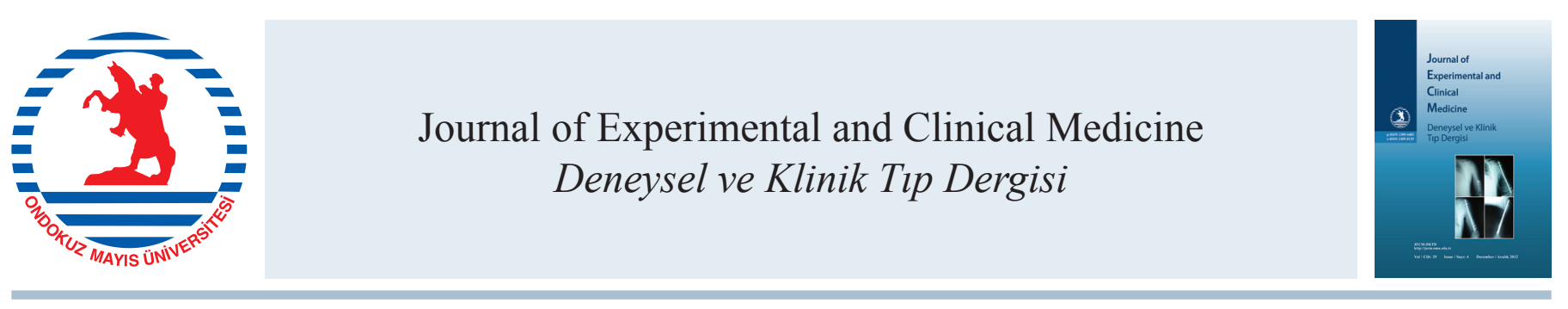

Klinik Araştırma / Clinical Research

doi: $10.5835 /$ jecm.omu.29.04.004

\title{
Santral seröz koryoretinopatide predispozan faktörler ve hastalığın doğal seyri
}

\author{
Predisposing factors in central serous chorioretinopathy and the natural progress of the illness
}

Yıldıray Yıldırım*, Fahrettin Akay

İzmir Asker Hastanesi, Göz Hastalıkları Kliniği, İzmir, Türkiye

\begin{tabular}{|c|c|}
\hline \multicolumn{2}{|c|}{ MAKALE BİLGÍLERİ } \\
\hline \multicolumn{2}{|c|}{ Makale geçmişi } \\
\hline Geliş tarihi & : 14 / 03 / 2012 \\
\hline Kabul tarihi & : $20 / 03$ / 2012 \\
\hline
\end{tabular}

\author{
* Yazışma Adresi: \\ Yildiray Yildırım \\ İzmir Asker Hastanesi \\ Göz Hastalıkları Kliniği \\ Hatay/İzmir \\ E-posta: doctoryyildirim@gmail.com
}

\author{
Anahtar Kelimeler: \\ Stres \\ Doğal seyir \\ Görme keskinliği \\ Kronikleşme \\ Etiyoloji
}

Santral seröz koryoretinopati

\section{Keywords:}

Central serous chorioretinopathy

Stress

Natural progress

Visual acuity

Chronicity

Etiology

\section{ÖZET}

Bu çalışmada santral seröz koryoretinopati (SSR)'li olgularda doğal seyir sonuçlarının ve predispozan faktörlerin bildirilmesi amaçlanmıştır. Primer SSR tanısı konulan ve 6 aylık takip boyunca herhangi bir tedavi almayan 34 olgu çalışmaya dahil edildi. Hastaların kayıtları retrospektif olarak incelendi. Tüm hastaların Snellen eşeline göre düzeltilmiş en iyi görme keskinlikleri, ön segment biomikroskopisi ve fundus muayeneleri, optik koherens tomografi ve fundus floresan anjiyografi tetkikleri kaydedildi. Kayıtlar SPSS 14,0 programı kullanılarak istatistiksel olarak değerlendirildi. Hastaların yaş ortalaması $33,17 \pm 6,13$, cinsiyetleri ise 26 erkek ve 8 bayand. Olguların \%58,8'inde A tipi kişilik mevcuttu. Hastalık başlamadan önce ciddi stres bozukluğu yaşayan hastaların oranı ise $\% 82,4(28)$ ' dür. Olguların mesleklere göre dağılımı ise \%35,3 askeri personel, \%20,6 polis, $\% 2,9$ doktor, $\% 11,8$ esnaf, $\% 23,5$ işçi, $\% 5,9$ ev hanımı olarak bulunmuştur. İlk başvuruda yapılan muayenede SSR gelişen gözdeki Snellen eşeline göre en iyi düzeltilmiş görme keskinliği ortalama $0,59 \pm 2,04$ 'dür. Hastalığın gözlere göre dağılım oranı ise; \%73,6 sağ göz, \%23,5 sol göz ve \%2,9 her iki göz olarak ortaya çıkmıştır. Hastalığın altı aylık takipten sonra kronikleşme oranı \%14,7'dir. Kronikleşmeyen olguların ortalama

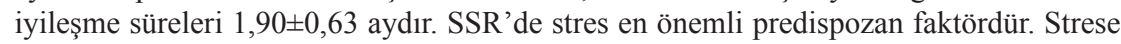
bağlı ortaya çıkan bazı biyokimyasal değişiklikler koroid damarlarında permeabilite değişikliği yapmaktadır. Hastalığın doğal seyrinde büyük bir kısmı spontan olarak iyileşmektedir. Kronik ve tekrarlayan olgularda tedavide bu patofizyolojik mekanizma düşünülerek ileri çalışmaların yapılması gerekmektedir.

J. Exp. Clin. Med., 2012; 29:272-275

\section{ABSTRACT}

To report the results of the natural progress and predisposing factors of the patients with central serous chorioretinopathy (CSR). The study comprised 34 patients diagnosed with primary central serous retinopathy and having no medication in preceding 6 months follow-up. Reports of the patients were examined retrospectively. The best corrected visual acuity (BCVA) with Snellen chart, results of anterior slit lamp microscopy and fundus examination as well as optical coherence tomography and fundus fluorescein angiography were evaluated. Statistical evaluation was performed with the SPSS program (SPSS 14.0 demo, SPSS inc., Chicago, Illinois). Mean age of the patients was $33.17 \pm 6.13$ years with 26 men and 8 women. A type personality was exist in $58.8 \%$ of total patients. The ratio of the patients having critical stress disorder before the illness had started was $82.4 \%$. Distribution of the patients according to their occupations were found as: $35.3 \%$ military personnel, $20.6 \%$ policeman, $11.8 \%$ trade, $23.5 \%$ worker and $5.9 \%$ housewife. On the first examination, mean BCVA of the eyes with CSR was $0.59 \pm 2.04$. The distribution ratio of the pathology according to the eyes were: $73.6 \%$ right eye, $23.5 \%$ left eye and $2.9 \%$ bilateral. The ratio of chronicity of the illness after 6 months follow-up period was $14.7 \%$. Mean recovery time of the patients with no chronicity was $1.90 \pm 0.63$ months. The most important predisposing factor in CSR is stress. Some biochemical changes due to stress leads to change the permeability of choroid capillaries. Most of the patients are recovering in the natural progression of the illness. Considering this physiopathological mechanism, further studies are necessary for the treatment of chronic and recurrent patients.

J. Exp. Clin. Med., 2012; 29:272-275 


\section{Giriş}

Santral seröz koryoretinopati (SSR) makula bölgesinde nöral retinadaki seröz dekolman olarak tanımlanır. Hastalık ilk olarak 1866 yılında Von Graffe tarafindan "makulanın tekrarlayan idiyopatik dekolmanı" olarak tanımlanmıştır. Maumenee bu tarihten 100 y1l sonra, florosan anjioskopi kullanarak hastalığın retina pigment epiteli (RPE) seviyesindeki sızıntıdan kaynaklandığını göstermiş ve hastalığa "santral seröz retinopati" adını vermiştir (Von Graefe, 1866; Maumenee, 1965). SSR etyolojisi genellikle idiyopatiktir. Yavaş başlayan ve gittikçe ilerleyen görmede bulanıklık, metamorfopsi ve mikropsi sık belirtilen şikayetlerdir (Robertson ve İllstrupp, 1983; Yanoff ve Duker, 2004). Hastalık en sık 20-50 yaş aras1 erkek populasyonda görülür. Fiziksel ve psikolojik stres yaşayan A tipi kişilik sahiplerinde daha sık rastlanmaktadır (Prunte ve Flammer, 1996). Vazokonstrüktif ajanlar, endojen hiperkortizolemi, sistemik kortikosteroid kullanımı, sigara alışkanlığ 1 , alkolizm, antihistaminikler ve psikofarmakolojik ajanların kullanımı ile birlikte SSR görüldüğü daha önceki yayınlarda rapor edilmiştir (Polak ve ark., 1995; Prunte ve Flammer, 1996). Bizim çalışmamızda ise SSR vakalarının doğal seyri ve predispozan faktörlerin bildirilmesi amaçlanmiştır.

\section{Gereç ve yöntem}

Ocak 2008 ve Aralık 2011 yılları arasında kliniğimize başvuran ve primer SSR tanısı konulan hastaların kayıtları retrospektif olarak incelendi. SSR tanısı klinik muayene bulgular1, optik koherens tomografi (OKT) ve fundus floresan anjiyografi (FFA) tetkikleriyle kondu. OKT'de seröz retinal elevasyon izlendi ve FFA'da SSR'ye tipik olan sızıntı görünümü tespit edilerek ayırıcı tanıda retinanın seröz dekolmanına yol açan inflamasyon, infiltrasyon ve koroidal neovaskülarizasyon ekarte edildi. Tüm hastaların Snellen eşeli ile düzeltilmiş en iyi görme keskinlikleri, ön segment biomikroskopik ve fundus muayene kayıtları değerlendirildi. Anamnez bulguları incelenerek, yakın zamanda önemli bir stres öyküsünün varlığı ve A tipi kişilik yapısı yönünden hastalar değerlendirilerek kaydedildi. A tipi kişilik genel olarak aceleci, yarışmacı, agresif ve stresli bir kişilik yapısı olarak tanımlanmaktadır. Bu bulgularla psikiyatri polikliniğine yönlendirilen hastalar ilgili uzman tarafından değerlendirildi ve kişilik testi Minnesota Multifaz Kişilik Envanteri (MMPI), ile tanıları doğrulandı. Düzenli olarak en az 6 ay boyunca takibi bulunan hastalar çalışmaya dahil edildi. Olgulardan daha önce sistemik kortikosteroid, antihistaminik, amfetamin ve antidepresan ilaç kullananlar çalışma dışı bırakıldı. Altı aylık takip boyunca herhangi bir tedavi almayan ve yukarıdaki özellikleri taşıyan 34 olgu rapor edildi. Verilerin SPSS 14.0(USA) programı kullanılarak analizi yapıldı. Bulgular ortalama \pm standart sapma (minimum-maksimum) şeklinde belirtildi.

\section{Bulgular}

Hastaların yaş ortalaması $33,17 \pm 6,13$ (21-45) yıl, cinsiyetleri ise 26 erkek $(\% 76,5)$ ve $8(\% 23,5)$ bayandır (Tablo 1$)$. Olguların \%58,8(n=20)'inde A tipi kişilik mevcuttur. Hastalık başlamadan önce stres bozukluğu yaşayan hastaların ora$\mathrm{n} 1$ ise $\% 82,4(\mathrm{n}=28)$ 'dür. Olguların mesleklere göre dağılımı ise $\% 35,3(\mathrm{n}=12)$ askeri personel, $\% 20,6(\mathrm{n}=7)$ polis, $\% 2,9$ $(n=1)$ doktor, \%11,8 $(n=4)$ esnaf, \%23,5 (8) işçi, \%5,9 (n=2) ev hanımı olarak bulunmuştur (Tablo 2). İlk başvuruda yapılan muayenede SSR gelişen gözdeki Snellen eşeline göre en iyi düzeltilmiş görme keskinliği ortalama $0,59 \pm 2,04(0,1$ $0,8)$ 'dür. Semptomların başlangıcından itibaren olguların ilk muayeneye gelme zamanları ortalama 2,20 1,09 (1-5) haftadır. Hastalığın gözlere göre dağılım oranı ise; \%73,5 (n=25) sağ göz, \%23,5 (n=8) sol göz ve \%2,9 (n=1) her iki göz olarak ortaya çıkmıştır (Tablo 3). Hastalığın altı aylık takipten sonra kronikleşme oranı \%14,7 (n=5) olarak bulundu. Kronikleșen olguların 3'ü askeri personel ve 2'si ise polis olarak tespit edildi. Kronikleşmeyen olguların ortalama iyileşme

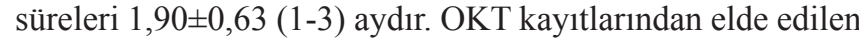
SSR'li gözlerdeki ilk başlangıç fovea kalınlıkları ortalama

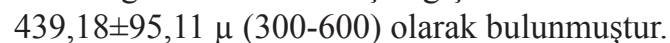

\begin{tabular}{lcc} 
Tablo 1. Olguların cinsiyetlerine göre dağılımı \\
Cinsiyet & Olgu sayısı & $\mathbf{\%}$ \\
\hline Erkek & 26 & 76,5 \\
Bayan & 8 & 23,5 \\
\hline
\end{tabular}

\begin{tabular}{lcc} 
Tablo 2. Olguların mesleklere göre dağılımı \\
Meslek & Olgu sayısı & $\mathbf{\%}$ \\
\hline Askeri personel & 12 & 35,3 \\
Polis & 7 & 20,6 \\
Doktor & 1 & 2,9 \\
Esnaf & 4 & 11,8 \\
İşçi & 8 & 23,5 \\
Ev hanımı & 2 & 5,9 \\
Toplam & 34 & 100
\end{tabular}

\section{Tablo 3. Olguların gözlere göre dağılımı}

\begin{tabular}{lcc} 
Göz & Olgu sayısı & $\mathbf{\%}$ \\
\hline Sağ göz & 25 & 73,5 \\
Sol göz & 8 & 23,5 \\
Her iki göz & 1 & 2,9 \\
Toplam & 34 & 100 \\
\hline
\end{tabular}

\section{Tartışma}

SSR oluşum patogenezinde fokal RPE sıvı kaçağı ile ilgili net bir bilgi oluşmamış olup subnöral retinal biriken sıv1nın koroid kaynaklı olduğu bilinmektedir. Prunte ve Flammer yaptıkları çalışmada, koroidin etkilenmiş kapiller lobülündeki anormal dolaşım sebebi ile iskemi oluştuğunu, sonrasında da artmış fokal hiperpermeabilite ile eksüdasyon geliştiğini, biriken sıvının RPE dekolmanı yaparak RPE hücreleri arasındaki s1kı bağlantıları kopararak kan-retina bariyerini bozduğunu bildirmişlerdir (Prunte ve Flammer, 1996). Bu teoriye göre iç koroidal tabakada gelişen primer patolojiden sonra retinanın sekonder olarak etkilendiği anlaşılmıştır. Primer SSR vakalarının doğal seyrinde büyük bir kısmının 3-4 ay içerisinde iyileştiği görülmüştür (Gass, 1977; Yannuzzi, 1986). Düşük oranda hastalık tekrar edebilir veya dekolman persistan olabilir. SSR altı aydan daha uzun sürerse kronik olarak tanımlanır (Yannuzzi ve ark., 1992).

SSR'de şimdiye kadar değişik tedavi seçenekleri denenmiştir. İlk olarak 1948'de psikoterapi önerilmiş ve daha sonra 
alfa adrenerjik blokörler, beta adrenerjik inhibitörler ve asetolazomid kullanılmıştır (Harrington, 1948; Gonzalez, 1992). Laser tedavi seçeneklerinden ise; retinal fotokoagülasyon (Burumcek ve ark., 1997), transpupiller termoterapi (TTT) (Shukla ve ark., 2006) denenmiştir. Günümüzde fotodinamik tedavi (FDT) (Cardillo Piccolino ve ark., 2003), anti-VEGF ajanları (Huang ve ark., 2009) ve kortikosteroid antagonistleri (ketokanazol) uygulanmaktadır (Meyerle ve ark., 2007).

Çalışmamızda SSR vakalarının büyük bir kısmının 6 ay içerisinde \%86,3 (29)'ünün tedavi almadan iyileştiği görülmüştür. Daha önce yapılan çalışmalar incelendiğinde olguların büyük kısmının 3-6 ay içerisinde spontan olarak tedavi almadan iyileştiği ve iyileşen olguların \%50'sinde nüks olduğu bildirilmiştir (Ficker ve ark., 1988; Castro-Correia ve ark., 1992). A-tipi kişilik yönünden şüpheli semptom ve bulgulara sahip hastalar psikiyatri uzmanı tarafından MMPI kişilik testine tabi tutularak değerlendirilmektedir. Bizim çalışmamızda olguların \%58,8 (20)'inde A tipi kişilik mevcuttur.

Psikolojik stres ile SSR ortaya çıkışında çok ciddi bir ilişki mevcuttur. Olguların \%82,4 ( $\mathrm{n}=28)$ 'ünde en önemli bir predispozan faktör olarak düşündüğümüz psikolojik stres mevcuttu. Olgulardaki psikolojik stres varlığı anamnezde hasta ifadelerinden tespit edilmiştir. Kişilik yapısı ve durumsal stresin patolojinin ortaya çıkışında çok önemli bir faktör olduğu diğer yapılan çalışmalarda ortaya konulmuştur (Zeligs, 1947). Hastaların yaş ortalaması $33,17 \pm 6,13(21-45)$ yıl olup büyük bir kısmı $(\% 76,5)$ erkek olarak bulunmuştur. Geniş vakalı yayınların demografik verileri incelendiğinde SSR'nin predominant olarak orta yaşı erkeklerde ve sosyal olarak topluma iyi entegre olmuş kişilerde rastlandığı açıklanmıştır (Quillen ve ark., 1996; Spahn ve ark., 2003).

Psikosomatik rahatsızlığın SSR'yi nasıl tetiklediği konusunda net bir bilgi yoktur. Emosyonel, fiziksel ve metabolik stresle beraber adrenal bezlerden glukokortikoid salınımını düzenleyen kortikotropin relasing hormon ve adrenokortikotropik hormonun hipotalamik sekresyonu artmaktadır (Chrousos ve Gold, 1992; Bouzas ve ark., 2002). A-tipi kişiliğe sahip olanlarda yapılan çalışmalarda plazma ve üriner kortizol sekresyonlarının arttığı gösterilmiştir. Aynı zamanda bu kişilik yapılarında stresli ortamlarda katekolamin seviyelerinin yüksek olduğu gösterilmiştir (Friedman ve ark., 1970; Pomerleau ve Pomerleau, 1990).

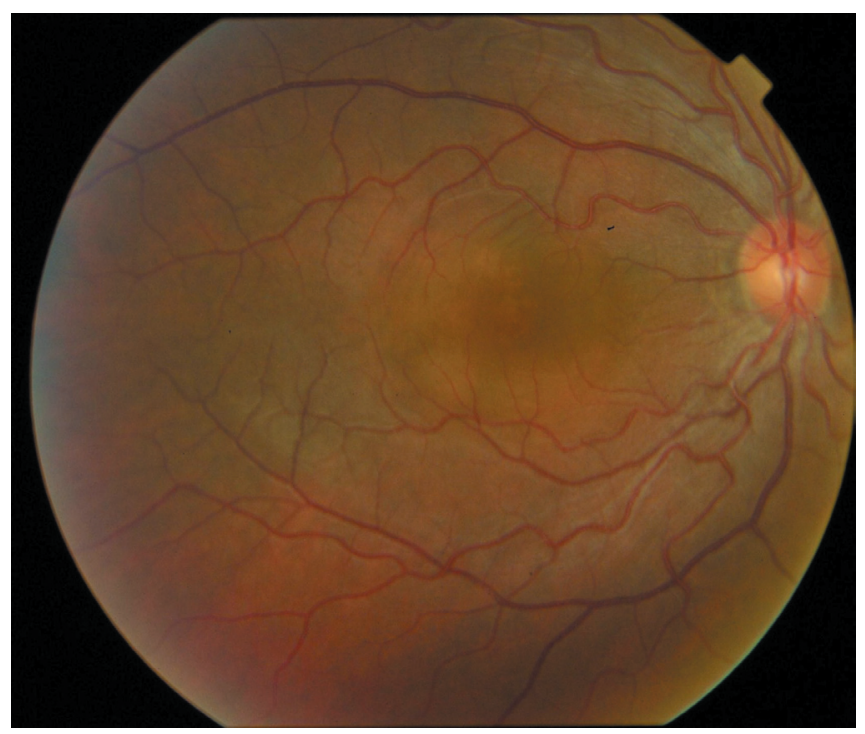

Şek. 1. SSR'de fundus görünümü
Hayvan modellerinde adrenalin, epinefrin ve diğer katekolaminler verilerek klinik ve anjiografik olarak SSR oluşturulmuştur (Nagayoski, 1971). Bizim çalışmamızda A-tipi kişiliğin ve stres faktörünün olguların büyük bir kısmında olması da bu patofizyolojik mekanizmayı desteklemektedir.

SSR tanısı konulmasında ve takibinde FFA ile OKT altın standart yöntemlerdir (Şek. 1, Şek. 2, Şek. 3 ve Şek. 4a,b). Çalışmamızda hastaların tanı ve takiplerinde bu yöntemler kullanılmıştır. OKT ile retinal kalınlığı ve subretinal sıvı kantitatif olarak ölçülür ve objektif olarak değerlendirilerek takip edilir (Hee ve ark. 1995; Costa ve ark., 2004).

Sonuç olarak SSR'de stres en önemli predispozan faktördür. Strese bağlı ortaya çıkan bazı biokimyasal değişiklikler koroid damarlarında permeabilite değişikliği yapmaktadır.

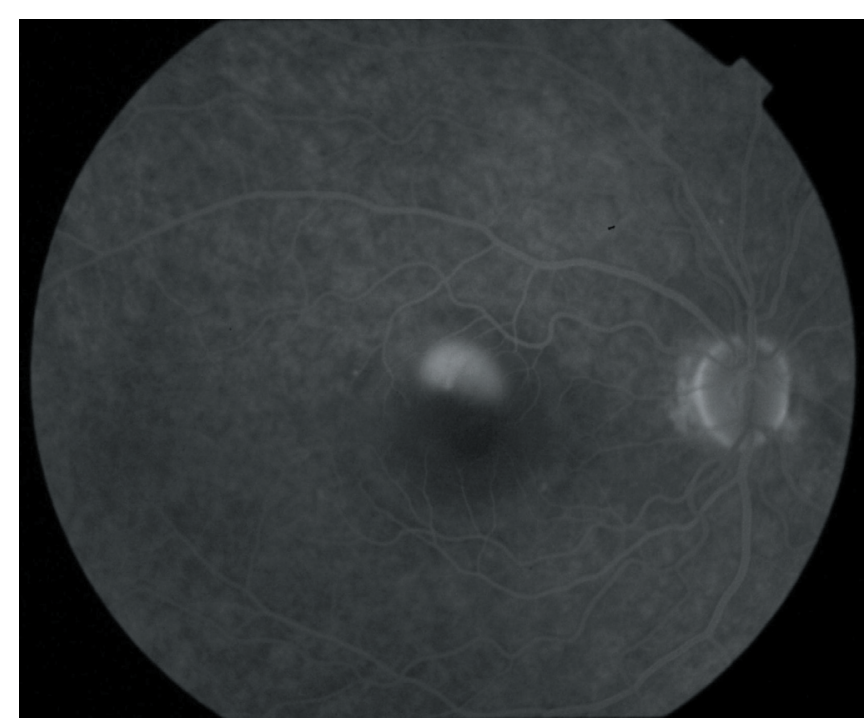

Şek. 2. FFA görüntülemesinde klasik sigara dumanı şeklinde izlenen subretinal sıvı birikimi.

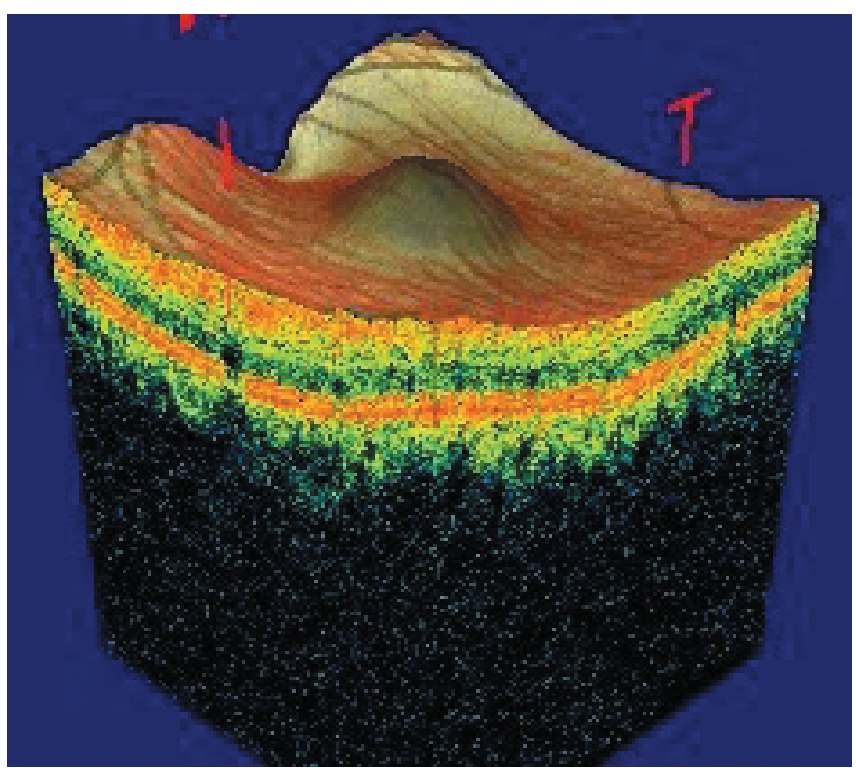

Şek. 3. SSR atağında OKT'de makulanın üç boyutlu görünümü.
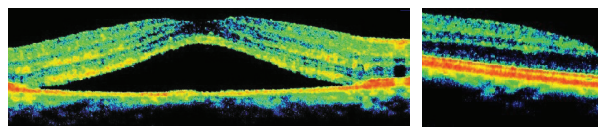

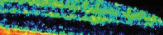

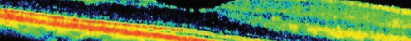

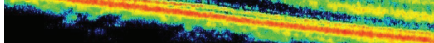

Şek. 4a. SSR atağında OKT'de makulanın kesitsel görünümü, b: Aynı hastanın iyileştikten sonra makulasının kesitsel görünümü. 
Hastalığın doğal seyrinde büyük bir kısmı spontan olarak iyileşmektedir. Kronik ve tekrarlayan olgularda tedavide bu patofizyolojik mekanizma düşünülerek ileri çalışmaların yapılması gerekmektedir.

\section{KAYNAKLAR}

Bouzas, E.A., Karadimas, P., Pournaras, C.J., 2002. Central serous chorioretinopathy and glucocorticoids. Surv. Ophthalmol. 47, 431-438.

Burumcek, E., Mudun, A., Karakocoglu, S., Arslan, M.O., 1997. Laser photocoagulation for persistent central serous retinopathy: results of long term follow-up. Ophthalmology. 104, 616-622.

Cardillo Piccolino, F., Eandi, C.M., Ventre, L., Rigault de la Longrais, R.C., Grignolo, F.M., 2003. Photodynamic therapy for chronic central serous chorioretinopathy. Retina. 23, 752-763.

Castro-Correia, J., Coutinho, M.F., Rosas, V., Maia, J., 1992. Long-term follow-up of central serous retinopathy in 150 patients. Doc. Ophthalmol. 81, 379-386.

Chrousos, G.P., Gold, P.W., 1992. The concepts of stress and stress system disorders. Overview of physical and behavioral homeostasis. JAMA 267, 1244-1252.

Costa, R.A., Calucci, D., Skaf, M., Cardillo, J.A., Castro, J.C., Melo, L.A.Jr, Martins, M.C., Kaiser, P.K., 2004. Optical coherence tomography 3: automatic delineation of the outer neural retinal boundary and its influence on retinal thickness measurements. Invest. Ophthalmol. Vis. Sci. 45, 2399-2406.

Ficker, L., Vafidis, G., While, A., Leaver, P., 1988. Long-term follow up of a prospective trial of argon laser photocoagulation in the treatment of central serous retinopathy. Br. J. Ophthalmol. 72, 829-834.

Friedman, M., Byers, S.O., Rosenman, R.H., Franklin, R. E., 1970. Coronary-prone individuals (Type-A behavior pattern). Some biochemical characteristics. JAMA. 212, 1030-1037.

Gass, J.D.M., 1977. Photocoagulation treatment of idiopathic central serous chorioretinopathy. Trans. Am. Acad. Ophthalmol. Otolaryngol. 83, 456-467.

Gonzalez, C., 1992. Decollements sereux retiniens. J. Fr. Ophthalmol. 15, 529-536.

Harrington, D.O., 1948. Psychosomatic interrelationship inophthalmology. Am. J. Ophthalmol. 31, 1241-1251.

Hee, M.R., Puliafito, C.A., Wong, C., 1995. Optical coherence tomography of central serous chorioretinopathy. Am. J. Ophthalmol. 120, 6574.

Huang, W.C., Chen, W.L., Tsai, Y.Y., Chiang, C.C., Lin, J.M., 2009. Intravitreal bevacizumab for treatment of chronic central serous chorioretinopathy. Eye. 23, 488-489.

Maumenee, A.E., 1965. Macular diseases: clinical manifestations. Trans. Am. Acad. Ophthalmol. 69, 605-613.

Meyerle, C.B., Freund, K.B., Bhatnagar, P., Shah, V., Yannuzzi, L.A., 2007. Ketoconazole in the treatment of chronic idiopathic central serous chorioretinopathy. Retina, 27, 943-946.

Nagayoski, K., 1971. Experimental study of chorioretinopathy by intravenous injection of adrenaline. Acta Soc. Ophthalmol. Jpn. 75, 17201727.

Polak, B.C.P., Baarsma, G.S., Snyers, B., 1995. Diffuse retinal pigment epithelyopathy complicating systemic corticosteroid treatment. Br. J. Ophthalmol. 79, 922-925.

Pomerleau, O.F., Pomerleau, C.S., 1990. Cortisol response to a psychological stressor and/or nicotine. Pharmacol. Biochem. Behav. 36, 211213.

Prunte, C., Flammer, J., 1996. Choroidal capillary and venous congestion in central serous choroidopathy. Am. J. Ophthalmol. 121, 26-34.

Quillen, D.A., Gass, D.M., Brod, R.D., Gardner, T.W., Blankenship, G.W., Gottlieb, J.L., 1996. Central serous chorioretinopathy in women. Ophthalmology. 103, 72-79.

Robertson, D.M., illstrupp, D., 1983. Direct, indirect and sham laser treatment in the management of central serous choroidopathy. Am. J. Ophthalmol. 95, 457-466.

Shukla, D., Kolluru, C., Vignesh, T.P., Karthikprakash, S., Kim, R., 2006. Transpupillary thermotherapy for subfoveal leaks in central serous chorioretinopathy. Eye. 22, 100-106.

Spahn, C., Wiek, J., Burger, T., Hansen, L., 2003. Psychosomatic aspects in patients with central cerous chorioretinopathy. Br. J. Ophthalmol. 87, 704-708.

Von Graefe, A., 1866. Kurzere abhandlungen, notizen und casaistische mitheilugnen vermischten inhalts: VI. Ueber zentrale recidivirende retinitis. Graefes Arch. Clin. Ophthalmol. 12, 211-215.

Yannuzzi, L.A., 1986. Type A behavior and central serous chorioretinopathy. Trans. Am. Ophthalmol. Soc. 84, 799-845.

Yannuzzi, L.A., Slakter, J.S., Kaufman, S.R., Gupta, K., 1992. Laser treatment of diffuse retinal pigment epitheliopathy. Eur. J. Ophthalmol. 2, 103-114.

Yanoff, M., Duker, J.S., 2004. Ophtalmology: 2nd edition, St. Louis: Mosby, p.p. 938-941.

Zeligs, M.A., 1947. Central angiospastic retinopathy: a psychosomatic study of the occurrence in military personnel. Psychosom. Med. 9, 110117. 